\title{
Feature similarity and feature bias in identification of visually presented letters
}

\author{
LINDA F. ALWITT* \\ Lee Terrace, Williamstown, Massachusetts 01267
}

\begin{abstract}
Reaction time (RT) to correct identification (ID) was measured for pairs of different letters presented on a memory drum. There were two types of lists. In Type I, visual and name similarity varied or thogonally at two levels. In Type II, one feature was constant at one level, while the other varied at two levels of similarity. For both types of lists, RT is a function of the feature that is more easily extracted from the stimulus. Relative visual and name modality biases were estimated, and name bias is relatively more salient than visual bias under these experimental conditions. Specific letters differ in the amount of feature processing required for correct ID and in the relative contribution of visual and name feature effects on this processing.
\end{abstract}

When a letter of the alphabet is identified, two of its features play a major role-its physical form and its name (Posner, Boies, Eichelman, \& Taylor, 1969; Cohen, 1969: Miller, 1972). Posner and his associates (1969, 1971) suggest that processing of the physical features takes place prior to processing of the name of a letter, when the letter is visually presented. Evidence from these studies supports models of memory that postulate a visual to verbal transformation in the analysis of symbolic stimuli (cf. Sperling, 1967; Atkinson \& Shiffrin, 1968). However, studies that manipulate feature modality rather than feature similarity suggest that processing of symbolic stimuli need not require a visual to verbal transformation and that there are separate auditory and visual storage systems (Scarborough, 1972a, b; Kroll, Parkinson \& Parks, 1972; Parkinson, 1972: Hopkins, Edwards, \& Gavelek, 1971). The type of storage system used depends, in part, on task expectations (Frost, 1972), task requirements (Tversky, 1969), and input modality (Kroll et al, 1972).

Recent research on the effect of different kinds of masks on identification of letters (e.g., Turvey, 1973; Henderson, 1972) suggests that the physical features of visually presented letters are encoded, or registered. first and that analysis of a letter for its "meaning." its associative and response-related functions for the $\mathrm{S}$, takes place in a second stage of processing. This second stage of processing in the service of identification. characterized by Konorski (1967) as "gnostic," can involve either visual or name features of the letters. The results of studies that manipulate feature similarity and those that manipulate feature modality can be reconciled within a framework of these two processing stages. Results of feature similarity studies that suggest prior processing for physical features of letters may reflect the first stage of processing. Results of studies of feature modality reflect the second. "gnostic," stage of

*I would like to thank R. O. Rouse and the Psychology Department of Williams College for use of their equipment and facilities and an anonymous reviewer for his thoughtful comments. processing. The current study is concerned with the relationship of visual and name features in identification (ID) of letters and with their influence by input and output modality. The task in this study, identification, requires results of gnostic as well as physical processing. It is likely that same-different tasks require primarily physical processing.

Modality factors can potentially influence the various postulated stages in ID processing: stimulus registration. encoding, feature analysis and comparison, decoding, and response articulation. Alwitt (in press) tachistoscopically presented pairs of letters that varied orthogonally in visual and name similarity for an oral identification response. Identification, measured by number of correct pairs of letters, was an inverse function of the similarity of one feature at a high similarity level of the other feature. However, at low visual similarity, ID was a direct function of name similarity, while at low name similarity, ID was not related to visual similarity levels. The results at high levels of similarity were interpreted as a reflection of encoding processes, i.e., the more readily encoded feature leads to feature analysis and transformation of the stimulus for storage. The results at low similarity levels were interpreted as a reflection of decoding of the stored stimulus for a response in the required modality. That is, items stored via feature similarity are retrieved via feature similarity, but the search process is from most similar to least similar. Thus, more similar items are more readily retrieved than are less similar items. If decoding is a function of both response modality and the successful encoding feature, the ideal condition for correct ID was the combination of low visual (input) and high name (output) similarities. This condition did indeed show more correct IDs.

The current study was designed to assess the relative contributions of visual and name features to ID processing and to replicate the earlier study using a different methodology. Pairs of letters were visually presented for $1 \mathrm{sec}$ to be identified by saying their names aloud. The dependent measure was reaction time. 
Table 1

Visual and Name Similarity Ratings and Levels of Letter Pairs

\begin{tabular}{|c|c|c|c|c|}
\hline \multirow{2}{*}{$\begin{array}{l}\text { Letter } \\
\text { Pair }\end{array}$} & \multicolumn{2}{|c|}{ Rating } & \multicolumn{2}{|c|}{ Similarity Level } \\
\hline & Visual & Name & Visual & Name \\
\hline BP & .116 & .318 & $\mathrm{H}$ & $\mathrm{H}$ \\
\hline BR & .199 & 1.946 & $\mathrm{H}$ & L \\
\hline BV & 2.630 & .426 & L & $\mathrm{H}$ \\
\hline BY & 2.630 & 1.861 & L & L \\
\hline GC & .204 & .072 & H & $\mathrm{H}$ \\
\hline GO & .648 & 2.017 & $\mathbf{H}$ & $L$ \\
\hline GJ & 1.796 & .456 & L & $\mathrm{H}$ \\
\hline GK & $2.5+5$ & 1.640 & L & $\mathrm{L}$ \\
\hline $\mathrm{EB}$ & .214 & .175 & $\mathrm{H}$ & $\mathrm{H}$ \\
\hline $\mathrm{EF}$ & .359 & 1.743 & $\mathrm{H}$ & L \\
\hline ED & 1.459 & .392 & L & $\mathrm{H}$ \\
\hline EQ & 2.571 & 1.846 & $\mathrm{~L}$ & L \\
\hline
\end{tabular}

Two types of lists were presented. In Type I, visual and name similarity varied orthogonally at two levels. In Type II. one feature was constant at one level, while the other varied at two levels. The purpose of presenting Type I and II lists was to examine the effect of altering the relative importance of similarity of one feature over the other feature.

\section{METHOD}

\section{Apparatus}

Typewritten uppercase letter pairs in elite type were presented via a Lafayette memory drum for a duration of $1 \mathrm{sec}$. with an interitem interval of $7 \mathrm{sec}$. The task was to identify the letters by saying their names aloud into a microphone placed about 3 in. from the mouth. Reaction time (RT) was recorded in milliseconds via a Hunter Klockounter controlled by a Lafayette voice kes. The memory drum was connected via an electronic relay to the clock. The clock started when the shutter of the memory drum raised to display the letter pair and was stopped by the initiation of the roiced names of the letters.

\section{Design}

Two types of lists were presented. Type I lists consisted of pairs composed of a base letter and another letter, chosen because of its degree of visual and name similarity to the base letter. Two levels of visual and name similarity were orthogonally varied to produce four pairs. The four different pairs in both left-right orders, as well as one example of the base letter paired with an asterisk, made up a block of nine pairs for this type of list. (The single base letter was included in order to encourage the $S$ to attend to both letters of the pair rather than guess the second letter.) Each list consisted of 10 randomized blocks, for a total of 90 items. Type II lists consisted of the same pairs of letters as in Type I lists. However, for each Type II list. one feature was held constant at one level of similarity while the other feature varied, yielding two different letter pairs for each Type II list. The two pairs, in both left-right orders, plus the base letter paired with an asterisk made up a block of five pairs. Each list of this type consisted of eight randomized blocks, for a total of 40 items. Since each of the two features was presented at two levels of similarity, there were four forms of Type II lists: (a) both letter pairs on a list showed high name similarity (HN constant): (b) both pairs showed low name similarity (LN constant): ( $\mathrm{cl}$ both pairs showed high visual similarity $(\mathrm{HV}$ constant): (d) both pairs showed low visual similarity (LV constant). There were three Type I and 12 Type II lists. one for each of three base letters on each type of list.

The three base letters used to construct the lists were E. B and $G$. The second letter of a pair was matched from a list of 48 pairs of letters that had been previously rated for visual and name similarity (see Alwitt, in press). Table 1 shows the letter pairs used in this study, values of visual and name similarity for each pair. and levels of visual and name similarity to which each pair was assigned.

\section{Procedure}

Each of four sessions for each $S$ was preceded by a 45 -item warm-up list of letter pairs that differed from those on the test lists. The first session for each $\mathrm{S}$ consisted of the warni-up list and three Type II lists. The other three sessions consisted of the warm-up list, one Type I list. and three Type II lists. The order of presentation of Type I and II lists within a session was counterbalanced across sessions and Ss. The session on which a given Base Letter by Type List was presented was partially balanced across Ss. Preceding presentation of each list on the memory drum. the $S$ was allowed unlimited time to examine a card containing all of the items to be presented on that list, and the card was visible during all trials.

\section{Subjects}

Eight students. whose ages ranged from 15 to 20 years. served as paid Ss for four sessions of about $1 \mathrm{~h}$ each on 4 different days. None of the $S s$ had participated previously in a similar experiment.

\section{RESULTS AND DISCUSSION}

Reaction time differences may be due to the time needed to pronounce a specific letter rather than to feature similarity relationships of the letters of a pair. Since RT was recorded to initiation of the first or left-hand letter of a pair. this hypothesis can be tested by an ANOVA of left-right letter order effects. RT to identification when the base letter was on the left and pronounced first $(547 \mathrm{msec})$ is significantly shorter than when the base letter was on the right and pronounced last $(571 \mathrm{msec})[\mathrm{F}(1,7)=15.69, \mathrm{p}<.01]$. In order to minimize RT differences due to differential letter pronounceability, only trials on which the base letter was on the left were analyzed.

The first block of each list and all incorrect IDs were omitted from analysis. Data points were log transformed in order to satisfy the assumption of homogeneity of variance for the ANOVAs but were transformed to raw RTs in tables and text. A three-way repeated measurements ANOVA was carried out on RTs to correct ID for Type I lists (Visual by Name Similarity by Base Letter), and a two-way repeated measurements ANOVA was carried out for each Type II list (Feature Similarity by Base Letter). For each Visual by Name Similarity condition, a multiple correlation analysis was carried out on RTs for combined base-letter lists, using a mean raw RT for each S on Type I and the appropriate two Type II lists. The error rate was .03 for Type I lists and .04 for Type II lists. The difference in errors between the two types of lists is not significant (sign test $t<1$ ). 


\section{Feature Effects in Type I Lists}

Table 2 shows the mean RTs for Type I and Type II lists. RT for high name similarity (HN) is longer than for low name similarity (LN) pairs $[\mathrm{F}(1,687)=7.42$. $p<.01]$. The main effect for visual similarity is not significant $[F(1,687)<1]$, but there is a significant interaction of Visual by Name Similarity $[F(1,687)=7.75, p<.01]$. This interaction supports the assumption underlying this study that letters of a pair are processed relative to each other.

In pairs with high visual similarity (HV), the RT for $\mathrm{HN}$ pairs is longer than for $\mathrm{LN}$ pairs $[\mathrm{t}(349)=4.06$, $\mathrm{p}<.001]$. In pairs with high name similarity $(\mathrm{HN}), \mathrm{RT}$ to correct ID is longer for high visual similarity pairs (HV) than for low visual similarity pairs (LV) $[\mathrm{t}(316)=2.08, \mathrm{p}<.05]$. In $\mathrm{LV}$ pairs, there is no difference in RT with name similarity levels and, in LN pairs, RT does not vary with visual similarity levels. Identification, then, is a function of the feature that is more easily or rapidly extracted from the (physically registered) stimulus. These results suggest that (a) ID processing at the gnostic level is not necessarily determined by the input modality but can be determined by the feature that is easier to analyze in terms of similarity, and (b) visual and name features can be processed simultaneously, at least at some stages, which agrees with the results of Scarborough (1972a, b).

Does one feature have an advantage over the other in the process of identification? In a comparison of Visual by Name Similarity combinations HV/LN and LV/HN, the RT for the more salient low-similarity feature should be shorter. There is no difference in RT to correct ID $[t(359)=1.74]$. Thus, the RT, which measures time needed for ID processing of two visually presented letters, does not differ with differences in relative visual and name similarity.

Let us assume that RT is a function of (a) visual and name similarities and (b) visual and name modality biases on input/output as well as on feature analysis processes. Feature bias is determined by input/output modality and task requirements, feature processing requirements, and idiosyncratic $S$ operating style. The effects of feature similarity and feature bias on RT can be expressed as:

$$
\begin{aligned}
R T= & \text { (visual similarity) (visual bias) } \\
& +(\text { name similarity) (name bias) }
\end{aligned}
$$

This equation does not separate input and output from feature analysis and comparison as Briggs and Swanson (1970) have done. It estimates the relative effects of visual and name biases on ID processing as a whole. Feature similarity can be estimated by similarity ratings where a low rating represents high feature similarity (see Table 1). The Visual by Name Combinations HV/LN and $\mathrm{LV} / \mathrm{HN}$ present the clearest choice of the most likely feature to be used for feature analysis on the basis of
Table 2

Mean RTs (Milliseconds) to Correct ID for Letter Pairs of Type I and Type II Lists

\begin{tabular}{lcccc}
\hline & \multicolumn{4}{c}{ Combination } \\
\cline { 2 - 5 } List & HV/HN & HV/LN & LV/HN & LV/LN \\
\hline Type I & 571 & 530 & 547 & 547 \\
Type II & & & & \\
HN Constant & 588 & -- & 566 & -- \\
LN Constant & -- & 544 & -- & 547 \\
HV Constant & 602 & 567 & -- & -- \\
LV Constant & -- & -- & 529 & 545 \\
\hline
\end{tabular}

similarity. Substituting similarity ratings and mean RTs for the $\mathrm{HV} / \mathrm{LN}$ and $\mathrm{LV} / \mathrm{HN}$ combinations, the relative visual bias (VB) and name bias (NB) can be estimated:

$$
\begin{aligned}
& \mathrm{HV} / \mathrm{LN}: .402 \mathrm{VB}+1.635 \mathrm{NB}=530 \\
& \mathrm{LV} / \mathrm{HN}: 1.962 \mathrm{VB}+.425 \mathrm{NB}=547
\end{aligned}
$$

Solution of these simultaneous equations yields $\mathrm{NB}=271$ and $\mathrm{VB}=216$, which suggests that name bias is relatively stronger than visual bias in ID processing under the experimental conditions used here. Note that, even though the RTs of the two simultaneous equations are not significantly different, the relative advantage of name over visual bias still holds because of the feature similarity relationships in the two equations.

Results of the current study agree with Alwitt (in press) at high similarity levels but show no effects at low levels for either feature. It is likely that the two studies differ at low similarity levels because there is a relatively greater emphasis on the name feature in the current study. An increase in the relative importance of name over visual features will no longer allow the prediction that $\mathrm{LV} / \mathrm{HN}$ is the "ideal" processing condition, as in the earlier study, because LV is not as likely to be the successful encoding feature. Thus, there will be no great difference between name similarity conditions at LV. There are several pieces of evidence for greater relative name bias in the current study. First, visual and name bias, estimated for the earlier study, are virtually the same $(\mathrm{VB}=.53$ and $\mathrm{NB}=.52)$. (Bias values of the two studies cannot be compared directly because of response measure differences.) Second. the earlier study emploved tachistoscopically presented, visually degraded stimuli. while the current study employed relatively long-duration memory-drum stimuli and a response spoken into a microphone. The experimental situation itself may have emphasized one modality over the other. thereby influencing the feature biases. Third. the ANOVA main effect for visual but not for name similarity is significant in the earlier study, while the ANOVA main effect for name but not for visual similarity is significant in the current study. Fourth. the relative levels of visual and name similurity ratings can be estimated and compared. Because of the wide range of 
Table 3

Simple, Multiple, and Partial Correlations and Beta Coefficients for Each Visual by Name Combination

\begin{tabular}{lcccc}
\hline & \multicolumn{4}{c}{ Combination } \\
\cline { 2 - 5 } Ifeasure* & HV/HN & HV/LN & LV/HN & LV/LN \\
\hline $\mathrm{I}_{\mathrm{IN}}$ & .93 & .89 & .94 & .97 \\
$\mathrm{I}_{\mathrm{IV}}$ & .87 & .89 & .94 & .93 \\
$\mathrm{I}_{\mathrm{NV}}$ & .90 & .78 & .82 & .88 \\
$\mathrm{I}_{\mathrm{I} . \mathrm{NV}}$ & .93 & .95 & .99 & .98 \\
$\mathrm{I}_{\mathrm{IN} . \mathrm{V}}$ & .71 & .69 & .85 & .83 \\
$\mathrm{I}_{\mathrm{IV} . \mathrm{N}}$ & .19 & .69 & .85 & .67 \\
$\mathrm{~b}_{\mathrm{N}}^{2}$ & .62 & .26 & .27 & .42 \\
$\mathrm{~b}_{\mathrm{V}}^{2}$ & .03 & .26 & .27 & .12 \\
\hline
\end{tabular}

*Subscripts for measures are: $I=$ Type $I, V=$ Type $I I-V$ constant, $N=T$ ype $I I-N$ constant.

similarity used and the differential effects on RT at different similarity levels, an appropriate index of relative feature similarity is:

$$
(\mathrm{V}-\mathrm{N}) /(\mathrm{V}+\mathrm{N})
$$

where $\mathrm{V}=$ visual similarity rating and $\mathrm{N}=$ name similarity rating. The index for the current study $(.05)$ is less than for the earlier study $(.10)$, a difference which tentatively suggests that relative feature similarity differs in the two studies $[\mathrm{t}(28)=1.84, \mathrm{p}<.10]$. These differences between the two studies suggest that visual and name bias for ID processing can be altered by the choice of stimuli and experimental manipulanda and instructions. It can also be influenced, most likely, by choice of input (cf. Kroll et al, 1972) and output modalities.

\section{Similarity Effects in Type II Lists}

The results for Type II lists, in which one feature is held constant at one of the two levels, parallel results for Type I lists (see Table 2). When both pairs on a list show high visual similarity, the RT to $\mathrm{HN}$ pairs is longer than to $\mathrm{LN}$ pairs $[F(1,263)=9.96, p<.01]$. When pairs on a list show high name similarity, RT to HV pairs is longer than to LV pairs but only at the .10 level of significance $[F(1.250)=3.02, p<.10]$. When pairs show low similarity on one feature, RT does not vary with the similarity level of the other feature.

An $S$ can adopt one of several strategies to Type II lists: (a) he can pay relatively more attention to the constant feature because it is reliable; (b) he can pay relatively more attention to the variable feature because it provides more information; (c) he can pay attention to either feature in the same way he does to pairs in Type I lists, because both features can be used for ID processing. Use of Strategy a would predict no RT differences between levels of the variable feature. The Type II results, though, do show differences in RT between the two levels of the variable feature when the constant feature is highly similar. Use of Strategy b would predict no RT differences between levels of the constant feature. RTs were compared for the two kinds of Type II lists on which the variable feature was the same: (a) HN constant vs LN constant when HV was the variable feature on both lists and when LV was the variable feature on both lists; (b) HV constant vs LV constant when $\mathrm{HN}$ was the variable feature on both lists and when $\mathrm{LN}$ was the variable feature on both lists. At the high levels of similarity of the variable feature, the RT to HN was greater than to $\operatorname{LN}[F(1,257)=8.05$, $\mathrm{p}<.01]$ and the RT to HV was greater than to LV $[F(1,255)=7.21, p<.01]$. At the low similarity levels of the variable feature, there was no difference in RT to high and low levels of the constant feature. These results suggest that the letter pairs in this study were ID processed independently of the list context. In effect. Ss appeared to use Strategy c. which would predict RT differences related to the feature from which it is easier to extract ID-relevant information.

\section{Relationship of Type I and Type II Lists}

Each letter pair on the Type I list is a member of two kinds of Type II lists. Although list context appears not to affect letter-pair identification, the relationship of the three lists can provide additional data on the effect of list context and on the relative importance of visual and name similarity in ID.

Table 3 shows the simple, multiple, and partial correlation coefficients and the squared beta coefficients for each Visual by Name Combination between Type I RTs and the appropriate pair of Type II RTs. For example, for $\mathrm{HV} / \mathrm{HN}, \mathrm{r}_{\mathrm{IN}}(.93)$ is the simple correlation between Type I RTs for $\mathrm{HV} / \mathrm{HN}$ and RTs for Type II HN-constant lists. Partial correlation coefficient $r_{I N}$.V (.71) is between Type I RTs and Type II HN-constant RTs, with Type II HV-constant RTs eliminated. The squared beta coefficient, $b_{N}^{2}(.62)$ is for the name-constant RTs.

The relative importance of visual and name features is evaluated on the basis of three types of evidence: (a) The savings of the variance due to r.NV $_{\text {I.Ner each of }}$ the simple rs should be smaller for the feature that is relatively more important; (b) the greater squared beta coefficient is that of the more important feature (McNemar, 1962); (c) the greater partial correlation coefficient is that of the more important feature.

The $\mathrm{N}$ - and V-constant Type II lists are virtually identical predictors for $\mathrm{HV} / \mathrm{LN}$ and for $\mathrm{LV} / \mathrm{HN}$ pairs. These results agree with results reported in the previous section: List context does not appear to affect letter-pair identification for these items. However, the Type II $\mathrm{N}$-constant lists appear to be somewhat better predictors than the V-constant lists of Type I item RTs for $\mathrm{HV} / \mathrm{HN}$ and for $L V / L N$. These results suggest that, for ambiguous pairs of letters, i.e., those with a similar 
degree of visual and name similarity, list context may influence the choice of the feature used in ID processing of the letters.

\section{Base Letter Effects}

Three base letters, E, B, and G, were paired with a second letter on the basis of feature similarity, but only one base letter list was presented to the $S$ at a time. Figure 1 shows the mean RTs to correct ID for each base letter in Type I lists. Base letter E differs from letters B and $G$ in several respects: (a) RT is faster to E pairs in virtually all conditions; (b) the visual similarity trend parallels B and G for HN pairs (see Fig. 1a); (c) the name similarity trend differs from that of $B$ and $G$ for $H V$ pairs (see Fig. 1b). The relatively short RT for G-pair $\mathrm{HV} / \mathrm{LN}$ is due to the specific letter paired with $\mathrm{G}$, which forms the common word GO.

The RT to identify pairs that contained base letter $\mathrm{E}$ on the left-hand side was about $60 \mathrm{msec}$ shorter than to identify pairs that contained B or $\mathrm{G}$ in both Type I $[t(703)=4.83, p<.001]$ and three Type II lists. In Type I lists, there is a significant interaction of Base Letters by Name Similarity $[F(2,687)=22.55$, $p<.001]$ and of Base Letters by Visual Similarity $[F(2,687)=3.77, \quad p<.05]$. In Type II lists, the interaction of Letters by Similarity is significant only for the HV-constant list: High name similarity E items are identified with a shorter RT than low name similarity E items $[\mathrm{t}(90)=1.98, \mathrm{p}<.05]$, while high name similarity $\mathrm{B}$ and $\mathrm{G}$ items are identified with a longer RT than low name similarity $B$ and $G$ items $[t(182)=7.14$, $p<.001]$. RT differences between $E$ pairs and $B$ and $G$ pairs are not a function of the relative proportions of visual and name similarity ratings: $E$ pairs are not different from B pairs $[\mathrm{t}(6)=1.33]$ or $G$ pairs $[\mathrm{t}(6)=1.66]$ on this index.

To the extent that the difference in RT between $E$ and $\mathrm{B}$ or $\mathrm{G}$ pairs is due to similarity (alternatively, it may be due to vowel vs consonant feature analysis), the explanation of these results resides in the name rather than the form of the base letters. First, if name analysis is based on the phonetic content of the letter name (Sperling \& Speelman, 1970; Parkinson, Parks, \& Kroll. 1971; Colegate \& Eriksen, 1970), then a biphonemic name will take longer to process than will a single phoneme. Although all three letters share the phoneme $[\mathrm{e}], \mathrm{B}$ and $\mathrm{G}$ each have an additional initial phoneme.

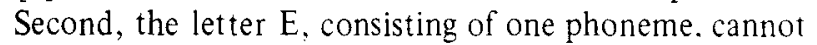
be confused on this basis with any other letter of the alphabet, but $B$ and $G$ share their second phoneme with seven other biphonemic letters of the alphabet. Third. the three base letters do not differ markedly in their visual feature confusability. A calculation of an average visual confusability index for each base letter with all other letters of the alphabet (except itself) from Townsend's (1971) nonmasked visual confusion matrix shows that the base letters are virtually the same: $\mathrm{E}=.026 . \mathrm{B}=.030 . \mathrm{G}=.026$.

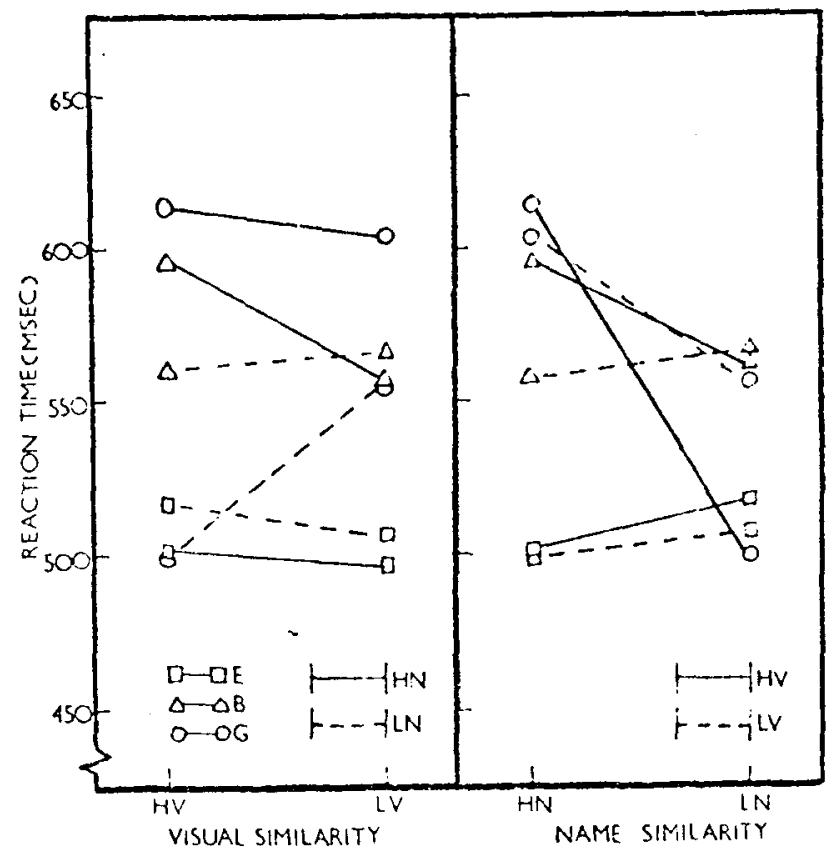

Fig. 1. Mean RTs for each base letter in Type I lists related to (a) visual similarity and (b) name similarity.

\section{CONCLUSIONS}

Several conclusions can be drawn from the results of this study. First, RT to correct ID is a function of the low-similarity feature, which is more easily extracted from the stimulus. This suggests that visual and name features of two letters can be processed simultaneously at some stage in ID processing. Second, feature similarity is one of several modality effects on letter ID. The other modality effects, characterized jointly as visual bias and name bias, do not necessarily serve the same end as does similarity. In the current study, name bias is relatively greater than is visual bias. This implies that visual similarity between two letters must be relatively more salient than name similarity to be used successfully as a feature analysis component. Third, visual and name biases can be manipulated by choice of stimuli, experimental manipulanda and instructions, by choice of input and output modalities, and possibly by list context for some items. Fourth, specific letters differ in the relative contribution of visual and name feature effects in ID processing. as well as in the amount of feature processing which presumably takes place. In this study. letter $E$ differs from $B$ and $G$ in amount and kind of ID processing required.

\section{REFERENCES}

Alwitt. L. F. Relationship of visual and name similarity of visually presented letters. Memory \& Cognition, in press.

Atkinson. R. C.. \& Shiffrin. R. M. Human memory: A proposed system and its control processes. In K. W. Spence and J. T. Spence (Eds.). The psychology of learning and motivation. Vol. 2. New York: Academic Press. 1968.

Briggs. G. L.. \& Swanson. J. M. Encoding. decoding, and central functions in human information processing. Journal of Lxperimental Psychology. 1970. 86. 296-308. 
Cohen. G. Some evidence for parallel comparisons in a letter recognition task. Quarterly Journal of Experimental Psychology. 1969. 21. 272-279.

Colegate. R. L.. \& Eriksen. C. W. Implicit speech as an encoding mechanism in visual perception. American Journal of Psycholog: 1970. 83, 208-215.

Frost. N. Encoding and retrieval in visual memory tasks. Journal of Experimental Psychology. 1972. 95. 317-326.

Henderson. L. Visual and verbal codes: Spatial information survives the icon. Quarterly Journal of Experimental Psychology: 1972. 24. 439-447.

Hopkins. R. H.. Edwards. R. E., \& Gavelek. J. R. Presentation modality as an encoding variable in short-term memory. Journal of Experimental Psychology. 1971. 90, 319-325.

Konorski. J. Integrative activitr of the brain. Chicago: University of Chicago Press. 1967.

Kroll. N. E. A., Parkinson, S. R., \& Parks, T. E. Sensory and active storage of compound visual and auditory stimuli. Journal of Experimental Psychology, 1972. 95. 32-38.

IcNemar. Q. Psvchological statistics. 3rd ed. New York: Wiler. 1962.

Miller. L. K. Letter-recognition: Effects of interitem similarity and report requirements. Perception \& Psychophysics, 1972. 11. 252.256

Parkinson, S. R. Short-term memory while shadowing: Multiple-item recall of visually and of aurally presented letters. Journal of Experimental Psychology. 1972, 92, $256-265$.

Parkinson. S. R.. Parks, T. E.. \& Kroll. N. E. A. Visual and auditory short-term memory: Effects of phonemically similar auditory shadow material during the retention interval.
Journal of Experimental Psychology, 1971, 87. 274-280.

Posner. M. I., Boies. S. J.. Eichelman. W. H.. \& Taylor. R. L. Retention of visual and name codes of single letters. Journal of Experimental Psychology. 1969, 79(3. Pt. 2). 1-16.

Posner, M. I.. \& Boies, S. J. Components of attention. Psychological Review, 1971. 78. 391-408.

Scarborough. D. L. Memory for brief visual displays of symbols Cognitive Psychology. 1972a, 3. 408-429.

Scarborough, D. L. Stimulus modality effects on forgetting in short-term memory. Journal of Experimental Psychology, 1972 b. $95,285-289$.

Sperling. G. Successive approximations to a model for a short-term memory task. Acta Psychologica, 1967. 27. 285-292.

Sperling. G., \& Speelman, R. G. A coustic similarity and auditory short-term memory: Experiments and a model. In D. A. Norman. Models of human memort. New York: Academic Press, 1970.

Townsend. J. T. Theoretical analysis of an alphabetic confusion matrix. Perception \& Psychophysics. 1971. 9. 40-50.

Turvey; M. T. On peripheral and central processes in vision: Inferences from an information-processing analy sis of masking with patterned stimuli. Psychological Review. 1973. 80. 1-52.

Tversky. B. Pictorial and verbal encoding in a short-term memory task. Perception \& Psychophysics. 1969. 6. 225-233.

(Received for publication January 13. 1973: revision received May 9. 1973.) 